\title{
Matryoshka of Special Democratic Forms
}

\author{
Chandrashekar Devchand $^{1}$, Jean Nuyts ${ }^{2}$, Gregor Weingart ${ }^{3}$ \\ 1 Institut für Mathematik der Universität Potsdam, Am Neuen Palais 10, \\ D-14469 Potsdam, Germany. E-mail: devchand@math.uni-potsdam.de \\ 2 Physique Théorique et Mathématique, Université de Mons-Hainaut, \\ 20 Place du Parc, B-7000 Mons, Belgium. E-mail: jean.nuyts@umh.ac.be \\ 3 Instituto de Matemáticas, Universidad Nacional Autónoma de México, \\ 62210 Cuernavaca, Morelos, Mexico. E-mail: gw@matcuer.unam.mx
}

Received: 6 January 2009 / Accepted: 3 August 2009

Published online: 2 October 2009 - (C) The Author(s) 2009. This article is published with open access at Springerlink.com

\begin{abstract}
Special $p$-forms are forms which have components $\varphi_{\mu_{1} \ldots \mu_{p}}$ equal to $+1,-1$ or 0 in some orthonormal basis. A $p$-form $\varphi \in \Lambda^{p} \mathbb{R}^{d}$ is called democratic if the set of nonzero components $\left\{\varphi_{\mu_{1} \ldots \mu_{p}}\right\}$ is symmetric under the transitive action of a subgroup of $\mathrm{O}(d, \mathbb{Z})$ on the indices $\{1, \ldots, d\}$. Knowledge of these symmetry groups allows us to define mappings of special democratic $p$-forms in $d$ dimensions to special democratic $P$-forms in $D$ dimensions for successively higher $P \geq p$ and $D \geq d$. In particular, we display a remarkable nested structure of special forms including a U(3)-invariant 2-form in six dimensions, a $\mathrm{G}_{2}$-invariant 3-form in seven dimensions, a $\mathrm{Spin}$ (7)-invariant 4-form in eight dimensions and a special democratic 6-form $\Omega$ in ten dimensions. The latter has the remarkable property that its contraction with one of five distinct bivectors, yields, in the orthogonal eight dimensions, the Spin(7)-invariant 4-form. We discuss various properties of this ten dimensional form.
\end{abstract}

\section{Introduction}

Special holonomy plays an important role in field theories. For instance, supersymmetry often requires that target manifolds have special holonomy. This property is also important for Yang-Mills theories. In dimensions greater than four, special holonomy offers the possibility of constructing solutions of the Yang-Mills equations satisfying the generalised self-duality equations first introduced for flat Euclidean spaces in [1] (see also [2-4]);

$$
\frac{1}{2} T_{m n p q} F_{p q}=\lambda F_{m n}, \quad m, n, \cdots=1, \ldots, d .
$$

Here, $F_{m n}$ are components of the curvature of a Yang-Mills connection $\nabla$ taking values in the Lie algebra of the gauge group and $T_{m n p q}$ are components of a 4 -form $T$. This 4-form acts as an endomorphism on the space of 2-forms. The curvature $F$ restricted to eigenspaces of $T$ corresponding to nonzero eigenvalues $\lambda$ satisfy the Yang-Mills equations $\nabla_{m} F_{m n}=0$ in virtue of the Bianchi identities $\nabla_{[m} F_{n p]} \equiv 0$. Interesting examples 
are the 4-forms invariant under $(\operatorname{Sp}(n) \otimes S p(1)) / \mathbb{Z}_{2}$, $\operatorname{Spin}(7)$ and $\mathrm{G}_{2}$ corresponding to Yang-Mills equations on quaternionic Kähler and exceptional holonomy manifolds (see e.g. [5-10]). Further examples of special holonomy structures are the $\mathrm{U}(n)$ invariant Kähler 2-forms in $2 n$ (real) dimensions and the $\mathrm{G}_{2}$ invariant Cayley 3-form in seven dimensions. It turns out that the latter forms are not only related to each other, but also to interesting higher rank forms in higher dimensions.

Recall that a constant $p$-form $\varphi$ in a $d$-dimensional Euclidean space is a calibration if for any $p$-dimensional subspace spanned by a set of orthonormalised vectors $e_{1}, \ldots, e_{p}$,

$$
\left(\varphi\left(e_{1}, \ldots, e_{p}\right)\right)^{2} \leq 1
$$

where equality holds for at least one subspace. Constant p-forms can always be rescaled to be calibrations. Many of the interesting calibrations which characterise special holonomy manifolds can be presented as special forms, all of whose nonzero components saturate the bound (1.2) (see Definition 1).

In this article, we wish to highlight relationships between special $p$-forms in $d$ dimensions and certain special $P$-forms $(P \geq p)$ in $D$ dimensions $(D \geq d)$, governed by discrete symmetries. Symmetric ways of embedding the $d$-dimensional space in the $D$-dimensional space leads us to a notion of democratic forms. We study examples, focusing our attention on specially interesting structures in dimensions seven, eight and ten. A remarkable nested structure, reminiscent of a matryoshka ${ }^{1}$, emerges in successively higher dimensions. In particular, this structure provides new examples of selfdualities.

\section{Special Forms, Symmetries and Democracy}

We concentrate on what we call special forms. Let $\left(e_{1}, \ldots, e_{d}\right)$ denote an orthonormal basis of $\mathbb{R}^{d}$.

Definition 1. A special p-form $\varphi$ is a p-form $\varphi \in \Lambda^{p} \mathbb{R}^{d}$ on $d$-dimensional Euclidean space $\mathbb{R}^{d}$ in the orbit under the special orthogonal group $\mathrm{SO}(d, \mathbb{R})$ of

$$
\varphi=\sum_{1 \leq \mu_{1}<\cdots<\mu_{p} \leq d} \varphi_{\mu_{1} \cdots \mu_{p}} e_{\mu_{1}} \wedge e_{\mu_{2}} \wedge \cdots \wedge e_{\mu_{p}}
$$

with $\varphi_{\mu_{1} \ldots \mu_{p}} \in\{-1,0,1\}$.

Hence, a $p$-form $\varphi$ is special if there exist $d$ orthonormal basis vectors $e_{\mu}, \mu=1, \ldots, d$ such that for any subset of $p$ vectors $e_{\mu_{1}}, \ldots, e_{\mu_{p}}$ we have

$$
\varphi_{\mu_{1} \ldots \mu_{p}}=\varphi\left(e_{\mu_{1}}, \ldots, e_{\mu_{p}}\right) \in\{-1,0,1\} .
$$

Trivial examples are the volume forms in $d$ dimensions, which provide the Hodgeduality operators mapping $p$-forms to $(d-p)$-forms. Further well-known examples are the $\mathrm{G}_{2}$-invariant Cayley 3 -form in seven dimensions defined by the structure constants of the octonions, and the Spin(7)-invariant 4-forms in eight dimensions (see Sects. 4.3 and 4.4).

The orbits of special $p$-forms under $\mathrm{SO}(d, \mathbb{R})$ or $\mathrm{O}(d, \mathbb{R})$ play a major role in the following. Clearly there are only a finite number of orbits of special $p$-forms parametrised

\footnotetext{
1 матрёшка, a nested Russian doll.
} 
by the components $\varphi_{\mu_{1} \ldots \mu_{p}} \in\{-1,0,1\}$ under these two groups. Note however that distinct sets of components may give rise to special $p$-forms in the same orbit, because the subgroups $\mathrm{SO}(d, \mathbb{Z}) \subset \mathrm{SO}(d, \mathbb{R})$ or $\mathrm{O}(d, \mathbb{Z}) \subset \mathrm{O}(d, \mathbb{R})$ map the special form $\varphi$ in Eq. (2.1) into a special form parametrised by different components. These groups are isomorphic to the semidirect product of the permutation group $S_{d}$ acting naturally on $d-1$ or $d$ copies of $\mathbb{Z}_{2}$, namely $\operatorname{SO}(d, \mathbb{Z}) \cong S_{d} \ltimes \mathbb{Z}_{2}^{d-1}$ or $\mathrm{O}(d, \mathbb{Z}) \cong S_{d} \ltimes \mathbb{Z}_{2}^{d}$. Thus, special $p$-forms which appear to be different may nevertheless be in the same orbit under $\mathrm{SO}(d, \mathbb{R})$ or $\mathrm{O}(d, \mathbb{R})$. The action of an element $\left(\sigma, \eta_{1}, \ldots, \eta_{d}\right) \in S_{d} \ltimes \mathbb{Z}_{2}^{d}$, on the components of $\varphi$ is given by

$$
\varphi_{i_{1} \ldots i_{p}} \mapsto \eta_{i_{1}} \ldots \eta_{i_{p}} \varphi_{\sigma\left(i_{1}\right) \ldots \sigma\left(i_{p}\right)},
$$

where $\eta_{i}^{2}=1, i=1, \ldots, d$. If $\pi(\sigma)$ is the parity of $\sigma$, the elements of the subgroup $\mathrm{SO}(d, \mathbb{Z}) \subset \mathrm{O}(d, \mathbb{Z})$ are those with $\eta_{1} \eta_{2} \ldots \eta_{d} \pi(\sigma)=1$. The orbit of a special $p$-form may always be labeled by a choice of a representative (2.2).

If a $p$-form $\varphi$ is special, then $-\varphi$ is obviously also special as is its Hodge dual $(d-p)$-form $\star \varphi$. For $p$ odd, $-\varphi$ is always in the $\mathrm{O}(d, \mathbb{R})$ orbit of $\varphi$; for instance using the parity transformation $e_{j} \mapsto-e_{j}, j=1, \ldots, d$. We note that forms which can be brought to the special form (2.1) by a rescaling are also interesting.

An alternative description of special forms was given in [11]. Oriented sets were defined as equivalence classes of finite, totally ordered sets up to even permutations, i.e. every set has two different orientations differing by a "sign". Thus the oriented subsets of $\{1, \ldots, d\}$ are in bijective correspondence to oriented coordinate subspaces $\mathbb{R}^{p} \subset \mathbb{R}^{d}$ via $s=\left\{\mu_{1}, \ldots, \mu_{p}\right\} \longmapsto e_{\mu_{1}} \wedge \ldots \wedge e_{\mu_{p}}$. A special $p$-form can be thought of as a function from the set of oriented subsets $\left\{\mu_{1}, \ldots, \mu_{p}\right\} \subset\{1, \ldots, d\}$ to $\varphi_{\mu_{1} \ldots \mu_{p}} \in\{-1,0,1\}$ with the property that the function's values on the two different orientations of the same subset differ by a sign. Consequently a special $p$-form is specified completely by either of the two sets $\mathcal{J}^{ \pm}$of oriented subsets $\left\{\mu_{1}, \ldots, \mu_{p}\right\} \subset\{1, \ldots, d\}$ with $\varphi_{\mu_{1}, \ldots, \mu_{p}}= \pm 1$. We will call the set $\mathcal{J}:=\mathcal{J}^{+} \cup \mathcal{J}^{-}$the support, $|\varphi|:=\frac{1}{2}|\mathcal{J}|$ the weight of $\varphi$. We denote by $\mu^{(a)}:=\left\{\mu_{1}^{(a)}, \ldots, \mu_{p}^{(a)}\right\}, a=1, \ldots,|\varphi|$, the elements in $\mathcal{J}^{+}$, i.e.

$$
\varphi=\sum_{a=1}^{|\varphi|} e_{\mu_{1}^{(a)}} \wedge \cdots \wedge e_{\mu_{p}^{(a)}} .
$$

Restricted to the $p$-dimensional subspace spanned by any $\left\{\mu_{1}, \ldots, \mu_{p}\right\}$ belonging to the set $\mathrm{J}^{+}$, the form $\varphi$ is equal to the $p$-dimensional volume form and hence its components are

$$
\varphi_{\mu_{1} \ldots \mu_{p}}=\epsilon_{\mu_{1} \ldots \mu_{p}},
$$

where $\epsilon$ is the completely antisymmetric tensor with $p$ indices. This means in particular that a special form $\varphi$ in $d$ dimensions has non-zero components given by

$$
\varphi_{\sigma(1) \sigma(2) \ldots \sigma(p)}=\epsilon_{12 \ldots p} \text { for all } \sigma \in H,
$$

where $H$ is an appropriate set of permutations $\sigma$ of the indices $\{1, \ldots, d\}$ which map $\{1, \ldots, p\}$ to $\left\{\mu_{1}, \ldots, \mu_{p}\right\} \in \mathcal{J}^{+}$.

We can define a metric on the vertex space $\mathcal{P}^{p}(d)$, the space of (unoriented) $p$-element subsets of $\{1, \ldots, d\}$, by $\operatorname{setting} \operatorname{dist}(s, \tilde{s})=p-|s \cap \tilde{s}|$ and visualise 
the restriction of this metric to the set $\mathcal{J}^{+}$by drawing a graph with labeled edges, the vertices correspond to the elements of $\mathrm{J}^{+}$, the edges run between vertices of distance strictly less than $p$ and are labeled by this distance [11]. The graph of a special $p$-form $\varphi$ does not completely specify the components $\varphi_{\mu_{1} \ldots \mu_{p}} \in\{-1,0,1\}$ up to the action of $\mathrm{O}(d, \mathbb{Z})$; we still need to specify some relative sign. Nevertheless the graph gives a very condensed way of encoding the characteristics of a special $p$-form. In particular it is a useful tool in calculating the bisymmetry group introduced below.

- We call a $p$-form $\varphi$ permutation symmetric under the action of some element $\sigma \in S_{d}$ if

$$
\varphi_{\sigma\left(i_{1}\right) \ldots \sigma\left(i_{p}\right)}=\kappa \varphi_{i_{1} \ldots i_{p}}, \quad\left\{i_{1}, \ldots, i_{p}\right\} \in \mathcal{J}^{+},
$$

with $\kappa=1$. The set of all such transformations is the permutation symmetry group of $\varphi, G_{r} \subset S_{d}$, where $r=\left|G_{r}\right|$, the order. If $\kappa=-1$, we call the permutation $\sigma$ a permutation antisymmetry. If there exists one such antisymmetry $\tau$, then there are $r$ antisymmetries $\sigma \tau$ with $\sigma \in G_{r}$ and the set $B=\left\{\sigma, \sigma \tau \mid \sigma \in G_{r}\right\} \subset S_{d}$ forms a group of order $2 r$, which we call the permutation bisymmetry group. The permutation symmetry group $G_{r}$ is an invariant subgroup and $B / G_{r}=\mathbb{Z}_{2}$.

- We call a $p$-form orthogonal symmetric under the action of an element $\left(\sigma, \eta_{1}, \ldots, \eta_{d}\right) \in S_{d} \ltimes \mathbb{Z}_{2}^{d}$ if

$$
\varphi_{\sigma\left(i_{1}\right) \ldots \sigma\left(i_{p}\right)}=\kappa \eta_{i_{1}} \ldots \eta_{i_{p}} \varphi_{i_{1} \ldots i_{p}}, \quad\left\{i_{1}, \ldots, i_{p}\right\} \in \mathcal{J}^{+},
$$

with $\kappa=1$. The set of all such transformations forms a group called the orthogonal symmetry group of $\varphi$, denoted by $\widehat{G}_{s} \subset S_{d} \ltimes \mathbb{Z}_{2}^{d}$, where $s=\left|\widehat{G}_{s}\right|$.

If $\kappa=-1$, the transformation $\left(\sigma, \eta_{1}, \ldots, \eta_{d}\right)$ is called an orthogonal antisymmetry. The union of orthogonal symmetries and orthogonal antisymmetries will be called the orthogonal bisymmetries.

- We denote by $L^{(a)} \subset G_{r}$ (or $L^{(a)} \subset \widehat{G}_{r}$ ) the permutation (or respectively orthogonal) stabiliser of the oriented set $\mu^{(a)} \in \mathcal{J}^{+}$as the set of $\sigma \in G_{r}$ (or $\widehat{G}_{r}$ ) such that $\left\{\sigma\left(\mu_{1}^{(a)}\right), \ldots, \sigma\left(\mu_{p}^{(a)}\right)\right\} \equiv\left\{\mu_{1}^{(a)}, \ldots, \mu_{p}^{(a)}\right\}$ up to an even permutation. We define the permutation (resp. orthogonal) stability group as

$$
L=\bigcap_{a=1}^{|\varphi|} L^{(a)} .
$$

For example, the $d$-dimensional completely antisymmetric tensor $\epsilon_{i_{1} \ldots i_{d}}$ has the alternating group $A_{d}$ as its permutation symmetry group and $B=S_{d}$, the symmetric group, as its permutation bisymmetry group which is also its orthogonal symmetry group. The stability group $L$ of the set of indices $\{1,2, \ldots, d\}$ of its sole nonzero component is $A_{d}$ itself. As a consequence, for a form $\varphi$, a symmetry (resp. antisymmetry) which is an even permutation in $S_{d}$ corresponds to a symmetry (resp. antisymmetry) of $\star \varphi$. On the other hand, a symmetry (resp. antisymmetry) of $\varphi$ which is an odd permutation corresponds to an antisymmetry (resp. symmetry) of $\star \varphi$.

Definition 2. A p-form with components $\varphi_{i_{1} i_{2} \ldots i_{p}}$ is called democratic if there is a transitive action of one of the permutation bisymmetry groups on $\{1, \ldots, d\}$. In other words, for any $i_{1}, i_{2} \in\{1, \ldots, d\}$ there exists at least one element $\sigma$ of one of the permutation bisymmetry groups such that $\sigma\left(i_{1}\right)=i_{2}$.

Hodge-duality is clearly a bijection amongst democratic forms. 
The interplay between two related $p$ - and $P$-forms in dimensions $d$ and $D$ respectively and the connections between their related symmetry and bisymmetry groups turns out to be an interesting subject of study. We shall use these in order to discuss the 'presentations' of some particularly interesting special forms and also to construct $P$-forms on $D$-manifolds from $p$-forms $(p \leq P)$ on $d$-manifolds $(d<D)$.

\section{Higher Dimensional Forms from Lower Dimensional Forms}

We consider interesting ways of constructing $P$-forms $\Phi$ in a $D$-dimensional space $\mathbb{R}^{D}$ from $p$-forms $\varphi$ on a $d$-dimensional subspace $\mathbb{R}^{d}$ of $\mathbb{R}^{D}$, with $P=p+b, D=d+a$ and $0 \leq b \leq a$. Let us embed $\mathbb{R}^{d}$ in $\mathbb{R}^{D}$ in such a way that the first $d$ basis vectors of $\mathbb{R}^{D}$ are identical to the basis vectors $e_{i}, i=1, \ldots, d$ of $\mathbb{R}^{d}$. We label the basis vectors of $\mathbb{R}^{D}$ by indices $i=1, \ldots, d, d+1, \ldots, D-b, D-b+1, \ldots, D$.

Given the components of a $p$-forms $\varphi \in \Lambda^{p} \mathbb{R}^{d}$ and an appropriate subgroup $H \subset S_{D}$, we may define a $P$-form $\Phi \in \Lambda^{P} \mathbb{R}^{D}$ with nonzero components given by

$$
\left\{\Phi_{\Sigma\left(i_{1}\right) \ldots \Sigma\left(i_{p}\right) \Sigma(D-b+1) \ldots \Sigma(D)}=\varphi_{i_{1} \ldots i_{p}} \mid i_{1}, \ldots, i_{p} \in\{1, \ldots, d\}, \Sigma \in H\right\},
$$

where the $\Sigma \in H$ satisfy the following

Compatibility condition:

Consider any two sets of indices $\left\{i_{1}, \ldots, i_{p}\right\}$ and $\left\{j_{1}, \ldots, j_{p}\right\}$ belonging to $\{1, \ldots, d\}$ such that $\varphi_{i_{1} \ldots i_{p}}=\varphi_{j_{1} \ldots j_{p}}$. If two permutations $\Sigma, \Sigma^{\prime} \in H$ have the property that

$$
\begin{aligned}
& \left\{\Sigma\left(i_{1}\right), \ldots, \Sigma\left(i_{p}\right), \Sigma(D-b+1), \ldots, \Sigma(D)\right\} \\
& \quad=\left\{\Sigma^{\prime}\left(j_{1}\right), \ldots, \Sigma^{\prime}\left(j_{p}\right), \Sigma^{\prime}(D-b+1), \ldots, \Sigma^{\prime}(D)\right\}
\end{aligned}
$$

then $\Sigma, \Sigma^{\prime}$ must be compatible in the sense that

$$
\Phi_{\Sigma\left(i_{1}\right) \ldots \Sigma\left(i_{p}\right) \Sigma(D-b+1) \ldots \Sigma(D)}=\Phi_{\Sigma^{\prime}\left(j_{1}\right) \ldots \Sigma^{\prime}\left(j_{p}\right) \Sigma^{\prime}(D-b+1) \ldots \Sigma^{\prime}(D)} .
$$

It is clear that the restriction of $\Phi$ to the $d$-dimensional subspace yields $\varphi$, i.e. $\left.\Phi\right|_{\mathbb{R}^{d}}=\varphi$, since the identity obviously belongs to $H$.

As we shall see in the next section, interesting cases arise when the forms are special and the subgroup $H$ is chosen so as to ensure democracy amongst the indices of $\Phi$. Particularly interesting examples correspond to the following three restrictions of (3.1):

A: For $D=d, P=p$, consider the non-zero components of a $p$-form $\Phi \in \Lambda^{p} \mathbb{R}^{D}$, with discrete symmetry group $G_{r}$ (see (2.7)). These are generated as the orbit under some subgroup $H \subset G_{r}$ of a set of $|\varphi| \leq|\Phi|$ given non-zero components, $\varphi_{\mu_{1}^{(a)} \ldots \mu_{p}^{(a)}}, a=1, \ldots,|\varphi|$, which define $\Phi$ thus:

$$
\Phi_{\sigma\left(\mu_{1}^{(a)}\right) \ldots \sigma\left(\mu_{p}^{(a)}\right)}=\varphi_{\mu_{1}^{(a)} \ldots \mu_{p}^{(a)}}, a=1, \ldots,|\varphi|, \sigma \in H \subset G_{r} .
$$

In other words, all the components of $\Phi$ arise from this formula. A presentation $P[m ; n](\Phi)$ of the $p$-form $\Phi$ is defined as the set of components $\varphi_{\mu_{1}^{(a)} \ldots \mu_{p}^{(a)}}=1$, $a=1, \ldots, m:=|\varphi|$ together with a set of $n$ permutations generating a presentation of $H$.

B: For $D>d, P=p$, a $p$-form $\varphi$ on $\mathbb{R}^{d}$ defines a $p$-form $\Phi$ on $\mathbb{R}^{D}$ with components

$$
\left\{\Phi_{\Sigma\left(i_{1}\right) \ldots \Sigma\left(i_{p}\right)}=\varphi_{i_{1} \ldots i_{p}} \mid i_{1}, \ldots, i_{p} \in\{1, \ldots, d\}, \Sigma \in H\right\},
$$

where $H$ is some subgroup of $S_{D}$. 
C: For $D=d+q, P=p+q$, a $p$-form $\varphi$ on $\mathbb{R}^{d}$ defines a $(p+q)$-form $\Phi$ on $\mathbb{R}^{d+q}$ with components

$$
\left\{\Phi_{\Sigma\left(i_{1}\right) \ldots \Sigma\left(i_{p}\right) \Sigma(d+1) \ldots \Sigma(D)}=\varphi_{i_{1} \ldots i_{p}} \mid i_{1}, \ldots, i_{p} \in\{1, \ldots, d\}, \Sigma \in H\right\}
$$

where $H$ is some subgroup of $S_{D}$.

\section{Examples}

Numerous examples of the constructions in (3.4), (3.5) and (3.6) can be generated, many rather trivial, e.g. the extension of a 1-form in one dimension to a 2 -form in two dimensions. However, relationships between $p$-forms invariant under the following subalgebras of $\mathfrak{s o}(D)$ provide interesting examples:

a) $\mathfrak{s o}(d) \subset \mathfrak{s o}(D), d<D$,

b) $\mathfrak{s u}(n) \oplus \mathfrak{u}(1) \subset \mathfrak{s o}(2 n)$,

c) $\mathfrak{g}_{2} \subset \mathfrak{s o}(7)$,

d) $\mathfrak{s p i n}(7) \subset \mathfrak{s o}(8)$.

In particular these examples fall into a remarkable nested structure of forms in successively higher dimensions up to eight. Furthermore, this matryoshka extends to interesting examples of special forms in ten dimensions.

4.1. $\mathfrak{s o}(d)$-invariant forms. The $\mathfrak{s o}(d)$-invariant $d$-forms clearly provide completely trivial examples. The unique nonzero component $\epsilon_{1 \ldots d}=1$, together with id $\in S_{d}$ provides a presentation. Moreover the $\mathfrak{s o}(d)$-invariant $d$-form $\epsilon$ can be extended to the $\mathfrak{s o}(D)$-invariant $D$-form $\epsilon$ for $D>d$ as follows:

$$
\epsilon_{\Sigma\left(i_{1}\right) \ldots \Sigma\left(i_{d}\right) \Sigma(d+1) \ldots \Sigma(D)}=\epsilon_{i_{1} \ldots i_{d}}, \quad \Sigma=\mathrm{id} .
$$

Clearly, the $d$-dimensional completely antisymmetric tensor $\epsilon_{i_{1} \ldots i_{d}}$ is fully democratic, the bisymmetry group $S_{d}$ acting transitively on its indices.

4.2. $\mathfrak{u}(n)$-invariant forms. Consider the $\mathfrak{u}(n)$-invariant 2-form $\omega$ on $\mathbb{R}^{2 n}$, with non-zero components

$$
\omega_{12}=\omega_{34}=\omega_{56}=\cdots=\omega_{(2 n-1)(2 n)}=1 .
$$

The vertex space $\mathcal{P}^{2}(2 n)$ consists of $n$ points. The permutation symmetry group of the 2-form $\omega$ is $G_{n !}=S_{n}$, the group of permutations of the $n$ ordered pairs of indices, $\{1,2\},\{3,4\},\{5,6\}, \ldots,\{(2 n-1),(2 n)\}$, generated by

$$
\begin{aligned}
& \sigma_{1}:=(13)(24)(5)(6) \cdots(2 n), \\
& \sigma_{2}:=\left(\begin{array}{llll}
13 & 3 & \cdots & 2 n-1
\end{array}\right)(246 \cdots 2 n) \text {. }
\end{aligned}
$$

The number of permutation antisymmetries is also $n$ !, generated by the composition of the generators in (4.3) with, for example, the permutation

$$
\tau_{1}:=\left(\begin{array}{ll}
1 & 2
\end{array}\right)(34)(56) \ldots(2 n-12 n) .
$$


There are $2^{n} n$ ! orthogonal symmetries and $2^{n} n$ ! orthogonal antisymmetries. These are either permutation symmetries or permutation antisymmetries multiplied by $2^{n}$ possible sign factors.

The 2-form $\omega$, as well as the $2 k$-forms $\varphi=\frac{1}{k !} \omega^{k}, k \leq n$, are democratic and special. For example, the permutation symmetry group of the 4-form $\frac{1}{2} \omega^{2}$ is the permutation bisymmetry group of $\omega$ and it has no antisymmetries.

Example 4.2.1. Starting from any one non-zero component, say $\omega_{12}$, the other components in (4.2) can be generated by the subgroup $H_{n} \subset G_{n}$ ! generated by $\sigma_{2}$. Hence a presentation is given by $P[1 ; 1](\omega)=\left\{\omega_{12} ; \sigma_{2}\right\}$. For $n=3$ the invariant subgroup $H_{3}$ is the commutator subgroup (the closure of the set of elements of the form $b^{-1} a^{-1} b a \forall a, b \in$ $\left.G_{6}\right)$.

Example 4.2.2. The 2-form $\omega \in \Lambda^{2} \mathbb{R}^{2 n}$ may be constructed from the 2-form in two dimensions, $\epsilon \in \Lambda^{2} \mathbb{R}^{2}$, with non-zero component $\epsilon_{12}=1$ thus:

$$
\omega_{\Sigma(a) \Sigma(b)}=\epsilon_{a b}, a, b=1,2, \Sigma \in\left\{\sigma_{2}^{m} \mid m=1, \ldots, n\right\}=H_{n} \subset G_{n !},
$$

where $H_{n}$ is the subgroup generated by $\sigma_{2}$ in (4.3).

4.3. The $\mathfrak{g}_{2}$-invariant form. Consider the $\mathrm{G}_{2}$ invariant special 3 -form $\psi$ on $\mathbb{R}^{7}$, with non-zero components $\psi_{a b c}$ given by any choice of the structure constants of the imaginary octonions. Let $\left\{e_{a}, a=1, \ldots 7\right\}$ denote the standard basis for $\operatorname{Im}(\mathbb{O}) \simeq \mathbb{R}^{7}$, with $e_{a} e_{b}=\psi_{a b c} e_{c}-\delta_{a b}$. A choice of the structure constants $\psi_{a b c}$ is given by

$$
\psi_{127}=\psi_{163}=\psi_{154}=\psi_{253}=\psi_{246}=\psi_{347}=\psi_{567}=1 .
$$

The vertex space $\mathcal{P}^{3}(7)$ consists of 7 points. The 7 -valent graph connecting these vertices has all edges labeled by distance 2 . The permutation symmetry group of $\psi$ (and naturally of its 4 -form dual $\star \psi$ ) is a group of order twenty-one, $G_{21}$, generated by the permutations

$$
\begin{aligned}
& \sigma_{3}:=(1254673), \\
& \sigma_{4}:=\left(\begin{array}{ll}
13 & 5
\end{array}\right)(246)(7) .
\end{aligned}
$$

The permutation $\sigma_{3}$ generates the commutator subgroup $H_{7} \subset G_{21}$. Since the permutation symmetry group includes an order 7 permutation, the form $\psi$ is manifestly democratic. There are no permutation antisymmetries $\tau$. Using Maple we have determined that the number of orthogonal symmetries is 672, with an order 168 commutator subgroup generated by either

$$
\{(157)(246)(3),(1265)(47)(3),(147)(253)(6)\}
$$

or, alternatively, by

$$
\{(14)(23)(5)(6)(7),(1247635),(134)(567)(2)\} .
$$

The number of orthogonal antisymmetries is also 672, obtained from the orthogonal symmetries by multiplying all the $\eta_{i}$ by -1 . 
Example 4.3.1. The component choice (4.6) can be generated from any non-zero component in (4.6) by the iterated action of $\sigma_{3}$. Thus a presentation is given by, for instance, $P[1 ; 1](\psi)=\left\{\psi_{127} ; \sigma_{3}\right\}$. Alternatively, a less economical presentation is given by $P[3 ; 1](\psi)=\left\{\psi_{127}, \psi_{136}, \psi_{246} ; \sigma_{4}\right\}$.

Example 4.3.2. The components of the 4-form dual $\star \psi \in \Lambda^{4} \mathbb{R}^{7}$ can be obtained in the following way:

$$
\star \psi_{\Sigma(1) \Sigma(2) \Sigma(3) \Sigma(4)}=\epsilon_{1234}, \Sigma \in H_{7} \subset S_{7}
$$

where $H_{7}$ is the group generated by the permutation $\sigma_{3}=(1254673)$.

Example 4.3.3. The 2-form $\omega$ in $\mathbb{R}^{6}$ given by (4.2) for $n=3$ affords an extension to the 3-form $\psi$ thus:

$$
\psi_{\Sigma(i) \Sigma(j) \Sigma(7)}=\omega_{i j}, i, j=1, \ldots, 6, \Sigma \in H \subset S_{7},
$$

where there are three types of 'minimal' choices of $H$, having only one generator:

- $H=H_{7}$ generated by $\sigma_{3}=(1254673)$,

- $H=H_{3}$ generated by (1)(265)(347),

- $H=H_{3}$ generated by (2)(136)(475).

Composing the mappings in Eqs. (4.5) and (4.12) immediately yields:

Example 4.3.4. Consider the two dimensional form $\epsilon \in \Lambda^{2} \mathbb{R}^{2}, \epsilon_{12}=1$. It yields the components (4.6) of the 3 -form $\psi$ in seven dimensions:

$$
\psi_{\Sigma(i) \Sigma(j) \Sigma(7)}=\epsilon_{i j}, i, j=1,2, \Sigma \in H_{7} \subset S_{7} .
$$

Choosing $\mathrm{H}_{7}$ to be the group generated by $\sigma_{3}=(1254673)$ again gives the set of $\psi$ 's in (4.6). In fact, choosing $\mathrm{H}_{7}$ to be the group generated by any seven-cycle of the form $(12 * * * 7 *)$ or $(12 * 7 * * *)$ provides equivalent choices of components of this 3-form.

This example yields a simple mnemonical construction of the structure constants of the imaginary octonions.

4.4. The $\mathfrak{s p i n}(7)$-invariant form. Consider the Spin(7)-invariant self-dual 4-form $\phi$ in $d=8$ [1] with non-zero components

$$
\begin{aligned}
1 & =\phi_{1234}=\phi_{1256}=\phi_{1278}=\phi_{1357}=\phi_{1386}=\phi_{1485}=\phi_{1476} \\
& =\phi_{2385}=\phi_{2376}=\phi_{2475}=\phi_{2468}=\phi_{3456}=\phi_{3478}=\phi_{5678}
\end{aligned}
$$

We note that each pair $(i, j)$ of indices occurs precisely thrice and the contraction with any 2-plane spanned by $\left\{e_{i}, e_{j}\right\}$ yields the $\mathfrak{u}(3)$-invariant 2-form (4.2) with $\left.\phi\left(e_{i}, e_{j}, \cdot, \cdot\right)\right|_{\mathbb{R}^{6}}=\omega$. The vertex space $\mathcal{P}^{4}(8)$ consists of 14 points. In the corresponding graph, every vertex is connected to 12 others by edges of distance 2 and to one antipodal point at distance 4 . This form is democratic and has a permutation symmetry group $G_{168}$ generated by

$$
\begin{aligned}
& \sigma_{5}:=(12)(367458), \\
& \sigma_{6}:=(8)(1254673) .
\end{aligned}
$$


The permutation $\sigma_{6}$ has a $1^{1} 7^{1}$ cycle decomposition. Its powers, apart from the identity, generate six independent permutations having the same cycle decomposition. There are 8 such permutations, corresponding to all 8 choices of the 1-cycle. In all they generate 48 permutations in the class $1^{1} 7^{1}$.

The permutation $\sigma_{5}$ has a $2^{1} 6^{1}$ cycle decomposition. Clearly, its inverse, $\sigma_{5}^{5}$, also. Further, $\sigma_{5}^{2}$ and $\sigma_{5}^{4}$ have $1^{2} 3^{2}$ cycles. There are 28 permutations of each of these four types, corresponding to all choices of the 2-cycle in $\sigma_{5}$. So, these generate 56 permutations in each of the classes $2^{1} 6^{1}$ and $1^{2} 3^{2}$. The third power, $\sigma_{5}^{3}$, generates a $2^{4}$ cycle. There are seven such permutations. Including the identity, we therefore have the $168=48+56+56+7+1$ elements of $G_{168}$.

The permutation $\sigma_{5}$ clearly decomposes into the product of the order 3 and order 2 permutations

$$
\begin{aligned}
\sigma_{7} & :=(1)(2)(357)(468), \\
\sigma_{8} & :=(12)(34)(56)(78),
\end{aligned}
$$

and a presentation for $G_{168}$ is given by $\sigma_{6}$ and $\sigma_{7}$.

The commutator subgroup of $G_{168}$ is the order 56 group generated by $\sigma_{6}$ and $\sigma_{8}$. It contains the 48 elements in the class $1^{1} 7^{1}$, the seven elements in the class $2^{4}$ and the identity.

The orthogonal symmetries of (4.14) total 10752 elements, with the commutator subgroup being the order 1344 group generated by (7)(1328456) and (6)(1572834). The form $\phi$ has no antisymmetries.

Example 4.4.1. The order 12 subgroup $H_{12} \subset G_{168}$ leaving the component $\phi_{1234}$ invariant is generated by $\sigma_{8}$ and

$$
\sigma_{9}:=(1)(6)(234)(587) \text {. }
$$

It has 14 left-cosets corresponding to the 14 components of $\phi_{i j k l}$, more precisely the action of the 14 cosets on $\phi_{1234}$ generates the 14 non-zero components of $\phi$.

The 4-form $\phi$ in (4.14) may be constructed in various ways from the $\mathfrak{s o}(n)-, \mathfrak{s u}(3) \oplus$ $\mathfrak{u}(1)$-, and $\mathfrak{g}_{2}$-invariant forms discussed above.

Example 4.4.2. Starting from the 4-form in $\mathbb{R}^{4}$ we can generate the 4-form $\phi$ in eight dimensions with components (4.14) thus:

$$
\phi_{\sigma(1) \sigma(2) \sigma(3) \sigma(4)}=\epsilon_{1234}, \sigma \in H_{12} \subset S_{8},
$$

where $H_{12}$ is the group generated by $\sigma_{8}$ and $\sigma_{9}$.

Example 4.4.3. From the components (4.6) of the $\mathfrak{g}_{2}$-invariant form $\psi \in \Lambda^{3} \mathbb{R}^{7}$, we may obtain the $\operatorname{Spin}(7)$-invariant 4-form $\phi$ :

$$
\phi_{\sigma(i) \sigma(j) \sigma(k) \sigma(8)}=\psi_{i j k}, i, j, k=1, \ldots, 7, \sigma \in H \subset S_{8} .
$$

By choosing $H$ appropriately, we obtain the components (4.14). Two possibilities are a) $H=H_{6}$ generated by $\sigma_{5}=(12)(367458)$, b) $H=H_{8}$ generated by the set $\{(12)(34)(56)(78),(13)(24)(57)(68),(15)(26)(37)(48)\}$. 
The $\phi_{m n p q}$ obtained this way satisfy the well-known relations (e.g. [1]),

$$
\phi_{i j k 8}=\psi_{i j k}, \quad \phi_{i j k l}=\frac{1}{6} \epsilon_{i j k l m n p} \psi_{m n p}, \quad i, \ldots, p=1, \ldots, 7 .
$$

Example 4.4.4. Analogously to (4.13), we may directly obtain the Spin(7)-invariant set of $\phi$ 's in (4.14) from $\epsilon_{i j}, \epsilon_{12}=1$, in two dimensions:

$$
\phi_{\sigma(i) \sigma(j) \sigma(7) \sigma(8)}=\epsilon_{i j}, i, j=1, \ldots, 2, \sigma \in H_{21} \subset S_{8},
$$

where $H=H_{21}$ is generated by (3)(1264758) and (1)(6)(234)(587).

\section{A $D=10$ Structure from a Spin(7) Structure in $d=8$}

5.1. Construction of a 6 -form in $D=10$ from a $\operatorname{Spin}(7)$-invariant 4 -form in $d=8$. Consider the Spin(7)-invariant self-dual 4-form $\phi_{m n p q}$ in $d=8$ given in (4.14). Its discrete symmetry group $G_{168}$ generated by the permutations (4.15) include the $\mathbb{Z}_{2} \times \mathbb{Z}_{2}$ transformations generated by the $2^{4}$ cycles

$$
\rho_{1}:=(18)(27)(36)(45), \quad \rho_{2}:=(14)(23)(58)(67) .
$$

Define the permutation $\sigma$ of $d$ indices, for $d$ even,

$$
\sigma:=(135 \ldots d-1)(246 \ldots d)
$$

which acts on the set of ordered pairs $\{1,2\},\{3,4\},\{5,6\}, \ldots$. We see that for $d=8$, this mapping squared, $\sigma^{2}=\rho_{1} \cdot \rho_{2}$.

We want to embed the form $\phi$ (4.20) into a form in $\mathbb{R}^{10}$ with orthonormal basis $\left(e_{n}\right)_{n=1, \ldots 10}$. For the components, we shall denote the $10^{\text {th }}$ index by a 0 . Clearly, a 6 -form $\Omega^{0}$ in ten dimensions which reduces to the above 4-form in eight dimensions may be defined in a trivial fashion by requiring the non-zero components to be given by

$$
\Omega_{m n p q 90}^{0}=\phi_{m n p q}, m, n, p, q=1, \ldots, 8,
$$

i.e. the 6-form $\Omega^{0}$ contracted with the volume form on the 9-10 plane yields the Spin(7)-invariant tensor (4.20). However, there is a less trivial possibility.

Consider a 6 -form $\Omega^{1}$ in $D=10$ with non-zero components

$$
\Omega_{\sigma(m) \sigma(n) \sigma(p) \sigma(q) 12}^{1}=\phi_{m n p q}, m, n, p, q=1, \ldots, 8 .
$$

We note that the components of $\Omega^{1}$ are compatible with the components of $\Omega^{0}$, in that

$$
\Omega_{m n p q 90}^{1}=\Omega_{m n p q 12}^{0}, m, n, p, q=3, \ldots, 8 .
$$

Similarly, the further 6-form having non-zero components

$$
\Omega_{\sigma^{2}(m) \sigma^{2}(n) \sigma^{2}(p) \sigma^{2}(q) 34}^{2}=\phi_{m n p q}, m, n, p, q \in\{1, \ldots, 8\} \backslash\{3,4\}
$$

is consistent with both $\Omega^{0}$ and $\Omega^{1}$, i.e.

$$
\begin{aligned}
& \Omega_{m n p q 34}^{0}=\Omega_{m n p q 12}^{2}, m, n, p, q=5, \ldots, 10, \\
& \Omega_{m n p q 34}^{1}=\Omega_{m n p q 90}^{2}, m, n, p, q=1,2,5, \ldots, 8 .
\end{aligned}
$$




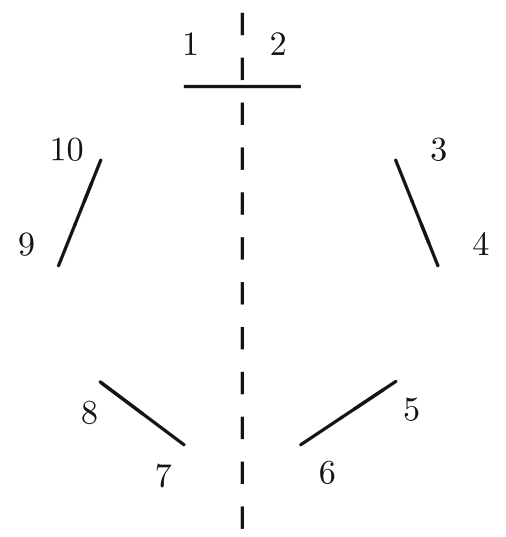

Fig. 1. The 6-form $\Omega$ is symmetric under $2 \pi / 5$ rotations generating a $Z_{5}$ symmetry. It is antisymmetric under reflections in the dotted line

In fact the five 6-forms $\Omega_{\sigma^{N}(m) \sigma^{N}(n) \sigma^{N}(p) \sigma^{N}(q) \sigma^{N}(9) \sigma^{N}(0)}^{N}, N=0, \ldots, 4$ are all compatible, allowing the definition of a 6 -form in ten dimensions manifestly invariant under the $\mathbb{Z}_{5}$ transformations between the five ordered pairs of indices in Fig. 1 generated by $\sigma=(13579)(24680)$, i.e.

$$
\{1,2\} \rightarrow\{3,4\} \rightarrow\{5,6\} \rightarrow\{7,8\} \rightarrow\{9,10\} \rightarrow\{1,2\} .
$$

This $\mathbb{Z}_{5}$-invariant 6-form has components given by

$$
\begin{gathered}
\Omega_{\sigma^{N}(m) \sigma^{N}(n) \sigma^{N}(p) \sigma^{N}(q) \sigma^{N}(9) \sigma^{N}(0)}=\phi_{m n p q}, \\
N=0, \ldots, 4, m, n, p, q=1, \ldots, 8 .
\end{gathered}
$$

Explicitly, for the choice (4.14), these are given by the 50 non-zero elements

$$
\begin{aligned}
& \Omega_{123456}=\Omega_{123478}=\Omega_{123490}=\Omega_{123579}=-\Omega_{123580} \\
& =-\Omega_{123670}=-\Omega_{123689}=-\Omega_{124570}=-\Omega_{124589}=-\Omega_{124679} \\
& =\Omega_{124680}=\Omega_{125678}=\Omega_{125690}=\Omega_{127890}=-\Omega_{134579} \\
& =\Omega_{134580}=\Omega_{134670}=\Omega_{134689}=\Omega_{135679}=-\Omega_{135680} \\
& =-\Omega_{135789}=\Omega_{135790}=\Omega_{136780}=-\Omega_{136890}=-\Omega_{145670} \\
& =-\Omega_{145689}=\Omega_{145780}=-\Omega_{145890}=\Omega_{146789}=-\Omega_{146790} \\
& =\Omega_{234570}=\Omega_{234589}=\Omega_{234679}=-\Omega_{234680}=-\Omega_{235670} \\
& =-\Omega_{235689}=\Omega_{235780}=-\Omega_{235890}=\Omega_{236789}=-\Omega_{236790} \\
& =-\Omega_{245679}=\Omega_{245680}=\Omega_{245789}=-\Omega_{245790}=-\Omega_{246780} \\
& =\Omega_{246890}=\Omega_{345678}=\Omega_{345690}=\Omega_{347890}=\Omega_{567890}=1 .
\end{aligned}
$$

The vertex space $\mathcal{P}^{6}(10)$ consists of 50 points, corresponding to a 49 -valent graph having two types of vertices: Type A vertices connected to 30 vertices at distance 2,16 vertices at distance 3 and 3 vertices at distance 4 and Type B vertices connected to 4 vertices at distance 1, 24 vertices at distance 2, 16 vertices at distance 3 and 5 vertices at distance 4. There are 10 vertices of Type A and 40 of Type B.

The symmetries of this democratic form are as follows. The permutation symmetry group is the order 60 alternating group $A_{5}$ of five elements, the five ordered pairs in (5.8). 
The number of permutation antisymmetries is also 60, obtained from the elements of the permutation symmetry group by multiplication by, for example, the reflection in the vertical axis of Fig. 1:

$$
\tau:=(12)(03)(94)(85)(76)
$$

There are 120 orthogonal symmetries and 120 orthogonal antiymmetries. The orthogonal symmetries which are not permutation symmetries have all their respective $\eta_{i}=-1$.

In the eight dimensional subspaces orthogonal to nonexceptional planes $\{a, b\}$, not in the set (5.8), this 6-form reduces to an SU(2)-invariant 4-form, which we discuss further in Sect. 5.3.

5.2. Self-duality. The six-form $\Omega$ given by (5.9) defines skew-symmetric endomorphisms on the space of 3 -forms, yielding generalised self-duality equations analogous to $(1.1)$,

$$
\frac{1}{6} g^{m_{4} n_{1}} g^{m_{5} n_{2}} g^{m_{6} n_{3}} \Omega_{m_{1} m_{2} m_{3} m_{4} m_{5} m_{6}} G_{n_{1} n_{2} n_{3}}=\lambda G_{m_{1} m_{2} m_{3}} .
$$

Its 4-form dual $\Phi=\star \Omega$ defines a symmetric endomorphism on the space of 2 -forms, satisfying equations of the form (1.1). To find the eigenvalues of a $2 k$-form $\Omega$ on $\Lambda^{k} \mathbb{R}^{D}$, we identify the components of the $k$-forms $G$ in $D$ dimensions,

$$
\left\{G_{m_{1} m_{2} \ldots m_{k}}, 1 \leq m_{1}<m_{2}<\cdots<m_{k} \leq D\right\}
$$

with the components of a vector in the $\left(\begin{array}{l}D \\ k\end{array}\right)$-dimensional space $\Lambda^{k} \mathbb{R}^{D}$ thus:

$$
G_{A}=G_{m_{1} m_{2} \ldots m_{k}} \text { where } A=1+\sum_{i=1}^{k}\left(\begin{array}{c}
m_{i}-1 \\
i
\end{array}\right)=1, \ldots,\left(\begin{array}{l}
D \\
k
\end{array}\right) .
$$

On this vector the $2 k$-form $\Omega$ may be represented as a $\left(\begin{array}{l}D \\ k\end{array}\right) \times\left(\begin{array}{l}D \\ k\end{array}\right)$ matrix,

$$
\Omega_{A B}=\Omega_{m_{1} \ldots m_{k} m_{k+1} \ldots m_{2 k}}, \quad A:=1+\sum_{i=1}^{k}\left(\begin{array}{c}
m_{i}-1 \\
i
\end{array}\right), \quad B:=1+\sum_{i=k+1}^{2 k}\left(\begin{array}{c}
m_{i}-1 \\
i
\end{array}\right) .
$$

In this notation, self-duality equations like (5.12) and (1.1) take the form of matrix equations allowing direct evaluation of the eigenvalues and eigenvectors using an algebraic computation programme like Maple or Reduce.

We find the characteristic polynomials for the 6-form (5.9) to be

$$
\left(\lambda^{6}+51 \lambda^{4}+699 \lambda^{2}+1369\right)^{4}\left(\lambda^{4}+42 \lambda^{2}+361\right)^{6}\left(\lambda^{2}+1\right)^{35}\left(\lambda^{2}+9\right)
$$

and that of its dual 4-form to be

$$
(\lambda+4)(\lambda+1)^{8}(\lambda-1)^{24}\left(\lambda^{2}+2 \lambda-19\right)^{6} .
$$

We have checked that the stability group $H^{\prime} \subset S O(10)$ of $\Omega$ (or equivalently $\Phi=\star \Omega$ ) has dimension 16 and is the direct product

$$
H^{\prime}=S U(4) \otimes U(1) \text {. }
$$


Under this stabilty group the $D=10$ dimensional vector module $V$ and the 45, 120 and 210 dimensional spaces of the two- three- and four-forms, respectively, decompose as

$$
\begin{aligned}
V=\mathbf{1 0}= & 4_{0}+\overline{4}_{0}+1_{+1}+1_{-1}, \\
\Lambda^{2} V=\mathbf{4 5}= & 15_{0}+6_{0}+6_{0}+4_{+1}+4_{-1}+\overline{4}_{+1}+\overline{4}_{-1}+1_{0}+1_{0}, \\
\Lambda^{3} V=\mathbf{1 2 0}= & 20_{0}+\overline{20}_{0}+15_{+1}+15_{-1}+\left(4_{0}\right)^{3}+\left(\overline{4}_{0}\right)^{3} \\
& +\left(6_{+1}\right)^{2}+\left(6_{-1}\right)^{2}+1_{+1}+1-1, \\
\Lambda^{4} V=\mathbf{2 1 0}= & 20_{0}^{\prime}+20_{+1}+20_{-1}+\overline{20}_{+1}+\overline{20}_{-1}+15_{0}+15_{0}^{\prime}+10_{0}+\overline{10}_{0} \\
& +\left(6_{0}\right)^{4}+\left(4_{+1}\right)^{2}+\left(4_{-1}\right)^{2}+\left(\overline{4}_{+1}\right)^{2}+\left(\overline{4}_{-1}\right)^{2}+\left(1_{0}\right)^{4},
\end{aligned}
$$

where the exponent denotes the multiplicity and the subscript the U(1) charge. We identify the eigenspaces in $\Lambda^{2} V$ of the 4-form $\Phi$ as follows:

$$
\begin{array}{ll}
\lambda=+1 & \Leftrightarrow 15+4+\overline{4}+1, \\
\lambda=-1 & \Leftrightarrow 4+\overline{4}, \\
\lambda=-4 & \Leftrightarrow 1, \\
\lambda=-1-2 \sqrt{5} & \Leftrightarrow 6, \\
\lambda=-1+2 \sqrt{5} & \Leftrightarrow 6 .
\end{array}
$$

The $\lambda=1$ eigenspace is the most interesting, satisfying a set of 21 equations amongst the 45 components of $\Lambda^{2} V$. The equations for the other eigenspaces are rather overdetermined. For the six-form, the roots of the $6^{\text {th }}$ order polynomial in (5.16) correspond to the 4's and $\overline{4}$ 's, the roots of the quartic correspond to the four 6's, the eigenspaces with $\lambda=i$ and $-i$ transform as $(20+15)$ and $(\overline{20}+15)$, respectively, and the two singlets have eigenvalues $\pm i \sqrt{3}$.

5.3. Reduction to nonexceptional planes. As we have seen, in the five exceptional eight dimensional embeddings in ten dimensions, the 6-form (5.10) reduces to the Spin(7)-invariant 4-form (4.14). Contracted with the bivectors spanning all the other planes, i.e. for $\{a, b\}$ not in the set of planes in (5.8), we obtain a 4-form with 17 nonzero components $T_{p q r s}$. For example in the space orthogonal to the $\{1,10\}$ plane we have

$$
\begin{aligned}
& T_{1256}=-T_{1678}=-T_{2356}=-T_{1357}=-T_{3467}=-T_{1458}=T_{2457} \\
& =-T_{2347}=T_{1247}=-T_{2567}=T_{3568}=-T_{1238}=T_{2578}=T_{3456} \\
& =-T_{2468}=T_{3478}=T_{1346}=1 .
\end{aligned}
$$

This 4-form in the eight dimensional space orthogonal to the $\{1,10\}$ plane is invariant under an $\mathrm{SU}(2)$ subgroup of $\mathrm{SO}(8)$. Under this $\mathrm{SU}(2)$ the 8-dimensional vector module of $\mathrm{SO}(8)$ decomposes as:

$$
\mathbf{8}=4[0] \oplus 2\left[\frac{1}{2}\right]
$$

i.e. four spin 0 modules and two spin $\frac{1}{2}$ modules. Here $[s]$ denotes the $2 s+1$ dimensional module of $\mathrm{SU}(2)$. The infinitesimal generators of $\mathfrak{s u}(2)$ are the following $8 \times 8$ matrices acting on the subspace $V^{2-9}$ spanned by the basis vectors $e_{2}, \ldots, e_{9}$ : 


$$
\begin{gathered}
T_{1}=\frac{1}{2 \sqrt{3}}\left(\begin{array}{cccccccc}
0 & 0 & 0 & 0 & 0 & 0 & 0 & 0 \\
0 & 0 & 0 & 1 & 0 & -1 & 0 & 0 \\
0 & 0 & 0 & 0 & 1 & 0 & -1 & 0 \\
0 & -1 & 0 & 0 & 0 & 1 & 0 & 0 \\
0 & 0 & -1 & 0 & 0 & 0 & 1 & 0 \\
0 & 1 & 0 & -1 & 0 & 0 & 0 & 0 \\
0 & 0 & 1 & 0 & -1 & 0 & 0 & 0 \\
0 & 0 & 0 & 0 & 0 & 0 & 0 & 0
\end{array}\right), \\
T_{2}=\frac{1}{2 \sqrt{3}}\left(\begin{array}{cccccccc}
0 & 0 & 0 & 0 & 0 & 0 & 0 & 0 \\
0 & 0 & 0 & 0 & 1 & 0 & -1 & 0 \\
0 & 0 & 0 & -1 & 0 & 1 & 0 & 0 \\
0 & 0 & 1 & 0 & -1 & 0 & 0 & 0 \\
0 & -1 & 0 & 1 & 0 & 0 & 0 & 0 \\
0 & 0 & -1 & 0 & 0 & 0 & 1 & 0 \\
0 & 1 & 0 & 0 & 0 & -1 & 0 & 0 \\
0 & 0 & 0 & 0 & 0 & 0 & 0 & 0
\end{array}\right), \\
T_{3}=\frac{1}{6}\left(\begin{array}{ccccccccc}
0 & 0 & 0 & 0 & 0 & 0 & 0 & 0 \\
0 & 0 & 2 & 0 & -1 & 0 & -1 & 0 \\
0 & -2 & 0 & 1 & 0 & 1 & 0 & 0 \\
0 & 0 & -1 & 0 & -1 & 0 & 2 & 0 \\
0 & 1 & 0 & 1 & 0 & -2 & 0 & 0 \\
0 & 0 & -1 & 0 & 2 & 0 & -1 & 0 \\
0 & 1 & 0 & -2 & 0 & 1 & 0 & 0 \\
0 & 0 & 0 & 0 & 0 & 0 & 0 & 0
\end{array}\right),
\end{gathered}
$$

satisfying the standard commutation relations $\left[T_{i}, T_{j}\right]=\epsilon_{i j k} T_{k}$. These $T_{i}$ 's clearly have nontrivial action on the subspace $V^{3-8}$ spanned by the basis vectors $e_{3}, \ldots, e_{8}$. In the eight dimensional space $V^{2-9}$ the vectors $(1,0, \ldots, 0),(0, \ldots, 0,1),(0,1,0,1,0,1$, $0,0)$ and $(0,0,1,0,1,0,1,0)$ are the four invariant vectors under the $\mathfrak{s u}(2)$ action. We find that the two spinor modules in (5.22) are spanned by the basis vectors

$$
\left\{b_{1}=(0,1+\sqrt{3} i, 0,1-\sqrt{3} i, 0,-2,0,0), b_{2}=(0,0, \sqrt{3}+i, 0,-\sqrt{3}+i, 0,-2 i, 0)\right\}
$$

and

$$
\left\{c_{1}=(0,0,1+\sqrt{3} i, 0,1-\sqrt{3} i, 0,-2,0), c_{2}=(0, \sqrt{3}-i, 0,2 i, 0,-\sqrt{3}-i, 0,0)\right\} .
$$

$b_{1}$ and $c_{1}$ are the eigenvectors of $T_{1}$ with eigenvalue $-i / 2$, whereas $b_{2}$ and $c_{2}$ are its eigenvectors with eigenvalue $i / 2$.

The above decomposition of the 8-dimensional vector module leads immediately to the following decomposition of the 28-dimensional space of 2-forms

$$
\mathbf{2 8}=9[0] \oplus 8\left[\frac{1}{2}\right] \oplus[1] .
$$

With the 4-form $T$ defined in (5.21), the characteristic polynomial of the self-duality equation (1.1) is

$$
\lambda^{4}\left(\lambda^{2}-5\right)\left(\lambda^{4}-8 \lambda^{2}+3\right)^{4}\left(\lambda^{6}-14 \lambda^{4}+33 \lambda^{2}-12\right) .
$$


Let us now discuss the association of the roots of (5.27) with the decomposition of the space of 2-forms (5.26) under $\mathfrak{s u}(2)$ :

- We identify the eight roots of $\left(\lambda^{2}-5\right)\left(\lambda^{6}-14 \lambda^{4}+33 \lambda^{2}-12\right)$, which are all distinct, with eight of the nine spin [0] states in (5.26).

- The four zero eigenvalues correspond to the ninth spin [0] state together with the spin [1] state.

- The remaining 16 eigenvalues, the roots of $\left(\lambda^{4}-8 \lambda^{2}+3\right)^{4}$, correspond to the spin $\left[\frac{1}{2}\right]$ states in the decomposition (5.26). Since there are only four distinct eigenvalues $\pm \lambda_{i}, i=1,2$ the corresponding eigenspaces transform as the four dimensional $\left[\frac{1}{2}\right] \oplus\left[\frac{1}{2}\right]$ representation.

\section{6. $\mathrm{SU}(4) \otimes \mathrm{U}(1)$-Invariant 4-Forms in Ten Dimensions}

As we see from the decomposition of the $210=\Lambda^{4}\left(4_{0}+\overline{4}_{0}+1_{+1}+1_{-1}\right)$, SU(4) $\otimes$ $\mathrm{U}(1)$ has four singlets. One of them arises from the tensor product $4 \otimes \overline{4} \otimes 1 \otimes 1$ and the other three singlets have their origin in $\Lambda^{4}\left(4_{0}+\overline{4}_{0}\right)$ and correspond to the three $\mathrm{SU}(4)$-invariant 4-forms discussed in Appendix B of [1]. Choosing complex coordinates $z_{1}=x_{1}+i x_{2}, z_{2}=x_{3}+i x_{4}, z_{3}=x_{5}+i x_{6}, z_{4}=x_{7}+i x_{8}, z_{5}=x_{9}+i x_{10}$, the four $\mathrm{SU}(4) \otimes \mathrm{U}(1)$-invariant forms may be expressed thus:

$$
\begin{aligned}
\Phi_{m n p q}^{A} & =\sum_{1 \leq i<j<k \leq 5} \epsilon_{m n p q z_{i}} \bar{z}_{i} z_{j} \bar{z}_{j} z_{k} \bar{z}_{k}, \\
\Phi_{m n p q}^{B} & =\sum_{\pi \in \mathbb{Z}_{5}} \epsilon_{m n p q z_{1} \bar{z}_{1} z_{2} \bar{z}_{2}\left(z_{3} \bar{z}_{4}+z_{4} \bar{z}_{5}+z_{5} \bar{z}_{3}\right)+\text { c.c. },} \\
\Phi_{m n p q}^{C}+i \Phi_{m n p q}^{D} & =\epsilon_{m n p q \bar{z}_{1} \cdots \bar{z}_{5}\left(z_{1}+\cdots+z_{5}\right)},
\end{aligned}
$$

where the sum in $\Phi^{B}$ is over all cyclic permutations of $(1, \ldots, 5)$. The corresponding vertex spaces $\mathcal{P}^{4}(10)$ consist of 10 points for $\Phi^{A}, 60$ points for $\Phi^{B}$ and 40 points for $\Phi^{C}$ and $\Phi^{D}$. The corresponding graphs have completely democratic vertices: For $\Phi^{A}$ every vertex is connected to 6 vertices at distance 2 and 3 vertices at distance 4 . For $\Phi^{B}$ every vertex is connected to 6 vertices at distance 1,27 vertices at distance 2,30 vertices at distance 3 and 6 vertices at distance 4 . For $\Phi^{C}$ and $\Phi^{D}$ every vertex is connected to 4 vertices at distance 1,18 vertices at distance 2, 12 vertices at distance 3 and 5 vertices at distance 4.

We find the characteristic polynomials of these 4-forms to be

$$
\begin{aligned}
\Phi^{A} & :(\lambda-1)^{20}(\lambda+1)^{24}(\lambda-4), \\
\Phi^{B} & :(\lambda+2)^{12}(\lambda-3)^{8}(\lambda-2)^{15}(\lambda+3)^{8}\left(\lambda^{2}+6 \lambda-36\right), \\
\Phi^{C}, \Phi^{D} & : \lambda^{33}\left(\lambda^{2}-20\right)^{6} .
\end{aligned}
$$

The duals of these 4-forms yield invariant 6-forms $\Omega^{A}, \Omega^{B}, \Omega^{C}$ and $\Omega^{D}$ having characteristic polynomials

$$
\begin{aligned}
\Omega^{A} & :\left(\lambda^{2}+1\right)^{55}\left(\lambda^{2}+9\right)^{5}, \\
\Omega^{B} & :\left(\lambda^{2}+36\right)^{1}\left(\lambda^{4}+60 \lambda^{2}+144\right)^{4}\left(\lambda^{2}+4\right)^{27}\left(\lambda^{2}+9\right)^{24}, \\
\Omega^{C}, \Omega^{D} & : \lambda^{80}\left(\lambda^{2}+20\right)^{20} .
\end{aligned}
$$


Table 1. Eigenvalues of the invariant 4-forms on the irreducible summands of $\Lambda^{2} V$

\begin{tabular}{llllllll}
\hline$\Lambda^{2} V=\mathbf{4 5}$ & $1_{0}$ & $1_{0}$ & $15_{0}$ & $4_{+1}+\overline{4}_{+1}$ & $4_{-1}+\overline{4}_{-1}$ & $6_{0}$ & $6_{0}$ \\
\hline$\Phi^{A}$ & -1 & 4 & -1 & 1 & -1 & 1 & 1 \\
$\Phi^{B}$ & $-3(1+\sqrt{5})$ & $-3(1-\sqrt{5})$ & 2 & 3 & -3 & -2 & -2 \\
$\Phi^{C}, \Phi^{D}$ & 0 & 0 & 0 & 0 & 0 & $2 \sqrt{5}$ & $-2 \sqrt{5}$ \\
\hline
\end{tabular}

Table 2. Eigenvalues of the invariant 6-forms on the irreducible summands of $\Lambda^{3} V$

\begin{tabular}{lllllllllll}
\hline$\Lambda^{3} V=\mathbf{1 2 0}$ & $20_{0}$ & $\overline{20}_{0}$ & $15_{ \pm 1}$ & $4_{0}^{2}$ & $\left(\overline{4}_{0}\right)^{2}$ & $4_{0}$ & $\overline{4}_{0}$ & $6_{+1}^{2}$ & $6_{-1}^{2}$ & $1_{ \pm 1}$ \\
\hline$\Omega^{A}$ & $i$ & $-i$ & $\pm i$ & $3 i$ & $-3 i$ & $i$ & $-i$ & $i$ & $-i$ & $\pm 3 i$ \\
$\Omega^{B}$ & $3 i$ & $-3 i$ & $\pm 2 i$ & $i(3 \pm \sqrt{21})$ & $-i(3 \pm \sqrt{21})$ & $3 i$ & $-3 i$ & $2 i$ & $-2 i$ & $\pm 6 i$ \\
$\Omega^{C}, \Omega^{D}$ & $i 2 \sqrt{5}$ & $-i 2 \sqrt{5}$ & 0 & 0 & 0 & 0 & 0 & 0 & 0 & 0 \\
\hline
\end{tabular}

The eigenvalues of the irreducible summands of these 4-forms and 6-forms are tabulated in Tables 1 and 2. The 4-forms $\Phi^{C}$ and $\Phi^{D}$ have identical eigenvalues. However, as endomorphisms of 2-forms, these do not commute.

The linear combination $-\Phi^{A}-\Phi^{C}$ is the dual of the 6-form $\Omega$ constructed in (5.9).

The 4-form $\Phi^{A}$ is the dual of the 6-form constructed in a similar fashion to (5.9) from the $S U(4) \otimes U(1) / \mathbb{Z}_{4}$-invariant 4-form in eight dimensions discussed in [1]:

$$
T_{m n p q}^{S U(4) \otimes U(1)}=\sum_{1 \leq i<j \leq 4} \epsilon_{m n p q z_{i}} \bar{z}_{i} z_{j} \bar{z}_{j}, m, n, p, q=1, \ldots, 8 .
$$

Using this to define a sixform as in (5.9), we obtain $\Omega^{A}=\star \Phi^{A}$. This clearly also has the special property that contracted with the volume form on any of the five exceptional planes in Fig. 1 yields the eight dimensional 4-form (6.10).

$\Phi^{D}$ is not invariant under the $\mathbb{Z}_{2}$ transformation indicated on Fig. 1. The four (SU(4) $\otimes$ $\mathrm{U}(1)$ )-invariants in ten dimensions are generated by the four dimensional centraliser of $S U(4) \otimes U(1)$ in $G L(10, \mathbb{R})$. In other words, acting on our invariant tensor (5.9) with the four-parameter set of global GL(10,R) transformations which commute with $\mathrm{SU}(4) \otimes$ $\mathrm{U}(1)$, yields the four-parameter set of $(\mathrm{SU}(4) \otimes \mathrm{U}(1))$-invariants above.

The four-forms $\Phi^{A}, \Phi^{B}, \Phi^{C}$ and $\Phi^{D}$ have permutation symmetry groups of order 240, 240, 120 and 120, respectively, all having $A_{60}$ as commutator subgroups. The number of permutation antisymmetries are $0,0,120,120$, the number of orthogonal symmetries, $122880=120 \cdot 2^{10}, 960,480,240$ and the number of orthogonal antisymmetries, $0,0,480$ and 240 , respectively.

\section{Comments}

Having seen that interesting 6-forms arise from the formula (5.9), the question arises whether there is a similarly constructed 8-form yielding interesting eigenvalue equations for a 4-form invariant under some subgroup $H^{\prime \prime} \subset \mathrm{SO}(\mathrm{d}+4)$.

The anwer is "yes" for $\Omega^{A}$ and $\Omega^{B}$ : We may construct consistent $\mathbb{Z}_{6}$-invariant 8-forms in 12 dimensions with components given by

$$
\Psi_{\sigma^{N}(m) \sigma^{N}(n) \sigma^{N}(p) \sigma^{N}(q) \sigma^{N}(r) \sigma^{N}(s) \sigma^{N}(11) \sigma^{N}(12)}=\Omega_{m n p q r s}^{I}, N=0, \ldots, 5,
$$


Table 3. Representative special 2-forms in 4 dimensions. The forms marked with $\mathrm{D}$ are democratic

\begin{tabular}{|c|c|c|c|c|c|c|c|c|c|}
\hline \multicolumn{10}{|c|}{ Representative forms for $p=2, d=4$} \\
\hline & $\epsilon_{12}$ & $\epsilon_{13}$ & $\epsilon_{14}$ & $\epsilon_{23}$ & $\epsilon_{24}$ & $\epsilon_{34}$ & $I_{1}$ & $I_{2}$ & \\
\hline $\bar{A}$ & 1 & 0 & 0 & 0 & 0 & 0 & -2 & 0 & \\
\hline$B_{1}$ & 1 & 1 & 0 & 0 & 0 & 0 & -4 & 0 & \\
\hline$B_{2}$ & 1 & 0 & 0 & 0 & 0 & 1 & -4 & 8 & $\mathrm{D}$ \\
\hline$B_{3}$ & 1 & 0 & 0 & 0 & 0 & -1 & -4 & -8 & $\mathrm{D}$ \\
\hline$C_{1}$ & 1 & 1 & 1 & 0 & 0 & 0 & -6 & 0 & \\
\hline$C_{2}$ & 1 & 1 & 0 & 0 & -1 & 0 & -6 & 8 & \\
\hline$C_{3}$ & 1 & 1 & 0 & 0 & 1 & 0 & -6 & -8 & \\
\hline$D_{1}$ & 1 & 1 & 1 & 1 & 0 & 0 & -8 & 8 & \\
\hline$D_{2}$ & 1 & 1 & 1 & -1 & 0 & 0 & -8 & -8 & \\
\hline$D_{3}$ & 1 & 1 & 0 & 0 & -1 & 1 & -8 & 16 & D \\
\hline$D_{4}$ & 1 & 1 & 0 & 0 & 1 & 1 & -8 & 0 & D \\
\hline$D_{5}$ & 1 & 1 & 0 & 0 & 1 & -1 & -8 & -16 & D \\
\hline$E_{1}$ & 1 & 1 & 1 & 1 & -1 & 0 & -10 & 16 & \\
\hline$E_{2}$ & 1 & 1 & 1 & 1 & 1 & 0 & -10 & 0 & \\
\hline$E_{3}$ & 1 & 1 & 1 & -1 & 1 & 0 & -10 & -16 & \\
\hline$F_{1}$ & 1 & 1 & 1 & 1 & -1 & 1 & -12 & 24 & D \\
\hline$F_{2}$ & 1 & 1 & 1 & 1 & 1 & 1 & -12 & 8 & D \\
\hline$F_{3}$ & 1 & 1 & 1 & -1 & -1 & -1 & -12 & -8 & D \\
\hline$F_{4}$ & 1 & 1 & 1 & -1 & 1 & -1 & -12 & -24 & D \\
\hline
\end{tabular}

for $I=A, B$. The corresponding 4-form duals have characteristic polynomials:

$$
\begin{aligned}
& \Phi^{A}:(\lambda+1)^{35}(\lambda-5)(\lambda-1)^{30}, \\
& \Phi^{B}:(\lambda-2)^{24}(\lambda+2)^{20}(\lambda-4)^{10}(\lambda+4)^{10}\left(\lambda^{2}+8 \lambda-80\right) .
\end{aligned}
$$

Acknowledgements. One of us (J.N.) thanks the Belgian Fonds National de la Recherche Scientifique for travel support, the Max-Planck-Institut für Mathematik in Bonn as well as Prof. Hermann Nicolai and the Max-Planck-Institut für Gravitationsphysik in Potsdam for hospitality.

Open Access This article is distributed under the terms of the Creative Commons Attribution Noncommercial License which permits any noncommercial use, distribution, and reproduction in any medium, provided the original author(s) and source are credited.

\section{A. Further Results: SO(d)-Invariants}

We denote by $I_{n}, n=1, \ldots, N(p, d)$, the independent $\operatorname{SO}(d, \mathbb{R})$-invariants constructed from a $p$-form $\varphi$, where $N(p, d)$ is the number of invariants.

Conjecture 1. The $S O(d, \mathbb{R})$ orbit of a special p-form is characterised by the values of the invariants.

This means that a general $p$-form is special if and only if the values of the invariants are equal to the values of the invariants of a representative special $p$-form, in whose orbit it then lies. If two $p$-forms have identical invariants, they belong to the same $\mathrm{SO}(d, \mathbb{R})$-orbit.

As an example we list in Table 3 the complete set of representative special 2-forms in 4-dimensions. The values of the two independent invariants

$$
\begin{aligned}
& I_{1}(\varphi)=\sum_{a, b} \varphi_{a b} \varphi_{b a}, \\
& I_{2}(\varphi)=\sum_{a, b, c, d} \epsilon_{a b c d} \varphi_{a b} \varphi_{c d}
\end{aligned}
$$


are given. If an arbitrary 2-form in 4-dimensions has values of $I_{1}, I_{2}$ appearing in the table, then it is in the orbit of the corresponding representative.

\section{References}

1. Corrigan, E., Devchand, C., Fairlie, D.B., Nuyts, J.: First order equations for gauge fields in spaces of dimension greater than four. Nucl. Phys. B214, 452-464 (1983)

2. Fairlie, D.B., Nuyts, J.: Spherically symmetric solutions of gauge theories in eight-dimensions. J. Phys. A17, 2867-2872 (1984)

3. Brihaye, Y., Devchand, C., Nuyts, J.: Selfduality for eight-dimensional gauge theories. Phys. Rev. D32, 990-994 (1985)

4. Devchand, C., Nuyts, J.: Super self-duality for Yang-Mills fields in dimensions greater than four. JHEP 12, 020 (2001)

5. Ward, R.S.: Completely solvable gauge field equations in dimension greater than four. Nucl. Phys. B236, 381-396 (1984)

6. Fubini, S., Nicolai, H.: The octonionic instanton. Phys. Lett. B155, 369-372 (1985)

7. Capria, M.M., Salamon, S.M.: Yang-Mills fields on quaternionic spaces. Nonlinearity 1, 517-530 (1988)

8. Nitta, T.: Vector bundles over quaternionic Kähler manifolds. Tohoku Math. J. 40, 425-440 (1988)

9. Tian, G.: Gauge theory and calibrated geometry. I. Ann. Math. 151(2), 193-268 (2000)

10. Alekseevsky, D.V., Cortes, V., Devchand, C.: Yang-Mills connections over manifolds with Grassmann structure. J. Math. Phys. 44, 6047-6076 (2003)

11. Devchand, C., Nuyts, J., Weingart, G.: Special graphs. Int. J. Geom. Meth. Mod. Phys. 3, 10111018 (2006)

Communicated by A. Connes 